\title{
Regulation of Chondrocyte Differentiation Level via Co-culture with Osteoblasts
}

\author{
RYUSUKE NAKAOKA, Ph.D., ${ }^{1}$ SUSAN X. HSIONG, M.S., ${ }^{2}$ and DAVID J. MOONEY, Ph.D. ${ }^{3}$
}

\begin{abstract}
The close apposition of osteoblasts and chondrocytes in bone and their interaction during bone development and regeneration suggest that they may each regulate the other's growth and differentiation. In these studies, osteoblasts and chondrocytes were co-cultured in vitro, with both direct and indirect contact. Proliferation of the co-cultured chondrocytes was enhanced using soluble factors produced from the osteoblasts, and the differentiation level of the osteoblasts influenced the differentiation level of the chondrocytes. In addition, the chondrocytes regulated differentiation of the co-cultured osteoblasts using soluble factors and direct contact. These data support the possibility of direct, reciprocal instructive interactions between chondrocytes and osteoblasts in a variety of normal processes and further suggest that it may be necessary to account for this signaling in the regeneration of complex tissues comprising cartilage and mineralized tissue.
\end{abstract}

\section{INTRODUCTION}

$\mathbf{R}$ EGENERATING TISSUES COMPRISING MULTIPLE CELL TYPES with specific and complex organization is a major goal of tissue-engineering studies. ${ }^{1}$ There are still numerous issues to be clarified before these types of tissues can be regenerated, including understanding the interaction between the different types of cells, controlling signaling cascades that regulate cell proliferation and differentiation, and regulating the spatial distribution of the various cell types. It has recently been reported that several tissues composed of multiple different types of cells can be regenerated by transplanting the various cell populations together on polymer scaffolds. ${ }^{2-4}$ For example, the co-transplantation of rat calvarial osteoblasts (RCOs) and bovine articular chondrocytes (BACs) on an appropriate carrier can lead to the formation of a growth plate-like tissue in severe combined immunodeficient (SCID) mouse. ${ }^{4}$ The RCO and BAC were randomly distributed inside the gels initially but reorganized to form clearly separated bony and cartilage- like tissues. The cartilage area of the tissue was composed of normal and hypertrophic chondrocytes, and the spatial localization of the different types of chondrocytes correlated to the location of the mineralized tissue. This finding suggested that there were interactions between the RCOs and BACs that induced hypertrophic differentiation of the BACs.

The growth plate is a highly organized structure composed of chondrocytes at different stages of differentiation between the epiphyseal and metaphyseal bone at the distal ends of the bone. Various growth factors and hormones, including fibroblast growth factors (FGFs), transforming growth factors (TGFs), bone morphogenetic proteins (BMPs), and parathyroid hormone-related protein (PTHrP), regulate the growth and structure of the growth plate. ${ }^{5} \mathrm{BMPs}$ have chondro-inductive effects on mesenchymal stem cells, and FGFs and insulin-like growth factor (IGF) have been reported to regulate chondrocyte proliferation, whereas TGF- $\beta$ and PTHrP inhibit chondrocyte maturation. ${ }^{5-8}$ BMP is also known to induce osteogenesis, and TGF- $\beta$ and FGFs

\footnotetext{
${ }^{1}$ Division of Medical Devices, National Institute of Health Sciences, Tokyo, Japan.

${ }^{2}$ Department of Chemical Engineering, University of Michigan, Ann Arbor, Michigan.

${ }^{3}$ Division of Engineering and Applied Sciences, Harvard University, Cambridge, Massachusetts.

This work was performed in the Department of Biologic and Materials Sciences, School of Dentistry, University of Michigan.
} 
are also recognized as important regulators of bone development and growth. ${ }^{9-13}$ We hypothesized that growth factors secreted by each cell type could regulate the formation of the growth plate-like tissue after implantation of gels containing RCOs and BACs. Because many of the relevant growth factors can be secreted from osteoblasts and chondrocytes, co-culture of osteoblasts and chondrocytes could potentially enhance chondrogenic differentiation as well as osteogenic differentiation. It has been reported that chondrocytes express soluble factors selectively promoting osteogenesis of mesenchymal stem cells. ${ }^{14}$ However, the effects of co-culture of osteoblasts on chondrocyte differentiation have not been reported.

The effect of $\mathrm{RCO}$ and BAC co-culture in vitro on the differentiation level of each cell type was investigated in these studies. RCOs and BACs were cultured alone or mixed in 12-well plates, and their differentiation level was determined qualitatively and quantitatively. To more directly assess the effects of soluble factors produced from RCOs or BACs on the other cell type, a transwell system was also used to co-culture RCOs and BACs while avoiding their direct contact.

\section{MATERIALS AND METHODS}

\section{Materials}

Medium, fetal calf serum (FCS), antibiotics, and other supplements for cell culture were purchased from Invitrogen Co. (Carlsbad, CA). All chemicals were purchased from Sigma (St. Louis, MO) unless stated. All reagents and primers for performing real-time polymerase chain reaction (PCR) were obtained from Applied Biosystems (Foster City, CA).

\section{Cell culture}

Primary RCOs were isolated from 1- to 2-day-old newborn Lewis rats (Harlan Sprague Dawley, Inc., Indianapolis, IN) and maintained in alpha-minimum essential medium (MEM) containing $10 \%$ FCS and $1 \%$ penicillinstreptomycin. ${ }^{4}$ Primary BACs were isolated from freshly slaughtered calf forelimbs and maintained in Dulbecco's modified Eagle medium (DMEM)/F12 (1:1) containing $10 \%$ FCS and $1 \%$ penicillin-streptomycin. ${ }^{4}$ The cells were maintained in incubators under standard conditions $\left(37^{\circ} \mathrm{C}\right.$, $5 \% \mathrm{CO}_{2}, 95 \%$ air, saturated humidity). The passage number of RCOs and BACs used in this study did not exceed 3 and 4 , respectively. Both cells were used for differentiation studies when sub-confluent.

\section{Co-culture study}

The RCOs, the BACs, and a mixture (RCO:BAC $=2: 1$ ) were seeded in 12-well plates at the total density of $9 \times 10^{4}$ / $2 \mathrm{~mL}$ medium/well using a 1:1 mix of alpha-MEM and
DMEM/F12 containing $10 \%$ FCS (the area cell density was $2.4 \times 10^{4} / \mathrm{cm}^{2}$ ). Some studies were performed using the medium containing $10 \mathrm{mM}$ beta-glycerophosphate, ascorbic acid $(50 \mu \mathrm{g} / \mathrm{mL})$, and $10^{-8} \mathrm{M}$ dexamethasone to determine the effects of RCO differentiation on BACs in the coculture. Cells were maintained in incubators under standard conditions. In all assays, culture media were changed once a week, and the cell numbers and differentiation levels assessed every 2 weeks. To determine whether the effect of RCOs and BACs on each other depended on direct cell-cell contact, a Transwell system (Corning Costar Co., Cambridge, MA) was also used for the co-culture experiments. RCOs and BACs were separately seeded into the wells or inserts and incubated for 4 weeks in those studies. The pore size and the area of an insert of the Transwell were $0.4 \mu \mathrm{m}$ and $1.1 \mathrm{~cm}^{2}$, respectively. The total cell number and the ratio of $\mathrm{RCO}$ and $\mathrm{BAC}$ seeded were kept similar to the simple co-culture system described above $\left(9 \times 10^{4} / 2 \mathrm{~mL}\right.$ medium/total well, RCO:BAC = 2:1). Therefore, the area densities of RCOs and BACs in the bottom well were $1.6 \times 10^{4}$ and $0.8 \times 10^{4} / \mathrm{cm}^{2}$, respectively. The area cell density in the insert well was adjusted to $2.7 \times 10^{4} / \mathrm{cm}^{2}$ when RCOs were cultured or to $5.4 \times 10^{4} / \mathrm{cm}^{2}$ when BACs were cultured in the bottom of the Transwell system.

The total cell number in each test well was determined using a Coulter counter (Coulter Electronics Inc., Hialeah, FL) after collecting cells. The cells were washed using phosphate-buffered saline (PBS) and detached by adding $0.5 \mathrm{~mL}$ of $0.05 \%$ trypsin solution and $0.5 \mathrm{~mL}$ of collagenase solution $(5 \mathrm{mg} / \mathrm{mL}$, F. Hoffmann-La Roche Ltd, Basel, Switzerland). After detachment, the cell number in the suspension was determined.

Differentiation levels of RCOs were estimated from changes in their alkaline phosphatase (ALP) activity, calcium deposition, and osteocalcin production. Total ALP activity of RCOs was estimated using the original procedure of Ohyama et al. ${ }^{15}$ Briefly, RCOs or co-culture were washed using PBS (-), followed by addition of $1 \mathrm{~mL}$ of $0.1 \mathrm{M}$ glycine buffer ( $\mathrm{pH} 10.5$ ) containing $10 \mathrm{mM}$ magnesium chloride, $0.1 \mathrm{mM}$ zinc chloride, and $4 \mathrm{mM}$ p-nitrophenylphosphate sodium salt. After incubating the cells at room temperature for $7 \mathrm{~min}$, the absorbance of the added buffer at $405 \mathrm{~nm}$ was detected using a microplate reader to evaluate ALP activity. Calcium deposition during the test period was qualitatively estimated using alizarin red staining. ${ }^{16}$ To determine osteocalcin production from the RCOs, their culture medium was replaced with fresh medium without FCS 1 day before the desired sampling, and the new medium was collected after $24 \mathrm{~h}$. The total amounts of osteocalcin in this medium were estimated using an osteocalcin enzyme-linked immunosorbent assay kit (Biomedical Technologies Inc., Stoughton, MA).

To determine effects of co-culture on the BAC differentiation level, Alcian blue staining was applied as a qualitative method to estimate production and deposition of proteoglycans and glycosaminoglycans from BACs. ${ }^{17}$ In 
Table 1. Sequences of Primers for Detection of Bovine Messenger Ribonucleic Acid Expression by Real-Time Polymerase Chain Reaction

\begin{tabular}{cll}
\hline GAPDH & Forward & TCCCCACTCCCAACGTGT \\
& Reverse & ATCTCATCATACTTGGCAGGTTTCT \\
Type II & Forward & GCATTGCCTACCTGGACGAA \\
collagen & Reverse & CGTTGGAGCCCTGGATGA \\
Type X & Forward & CCTCTTTCTCAGGATTCTTGGTG \\
collagen & Reverse & TTCAAGCAGATTTGTGTTAGCTCG \\
Aggrecan & Forward & GAGTGGAACGATGTCCCATGT \\
& Reverse & GCATTGATCTCGTATCGGTCC \\
\hline
\end{tabular}

some cases, proteoglycan-bound dye was extracted overnight with $4 \mathrm{M}$ guanidine hydrochloride at $4^{\circ} \mathrm{C}$, and the quantity determined from absorbance at $600 \mathrm{~nm} .{ }^{18,19}$ For quantitative analysis, a real-time PCR technique was applied. Briefly, total ribonucleic acid (RNA) from BACs was collected using the Absolutely RNA Microprep kit (Stratagene, La Jolla, CA). After its reverse transcription, realtime PCR was performed to detect expression of aggrecan,
type-II and type-X collagen messenger RNA (mRNA) using ABI Prism 7700 (Applied Biosystems). To normalize the data, mRNA expression of a housekeeping gene, glyceraldehyde-3-phosphate dehydrogenase (GAPDH), was also determined. Sequences of primers for the mRNA are shown in Table 1.

Gap junctional intercellular communication (GJIC) between cells was analyzed using fluorescene-activated cell sorting (FACS) according to Dürig et al. ${ }^{20}$ Briefly, one cell type, stained with calcein, was seeded in a culture dish containing the other cell type that had already been stained with the PKH 26 fluorescent dye. After incubation, all cells were trypsinized to prepare a cell suspension and subjected to FACS analysis to determine the ratio of exhibiting fluorescence from both markers (PKH 26 and calcein) as an estimate of the GJIC level between the 2 cell types.

\section{STATISTICS}

All data were expressed as mean \pm standard deviation. The Fisher-Tukey criterion was used to control for multiple

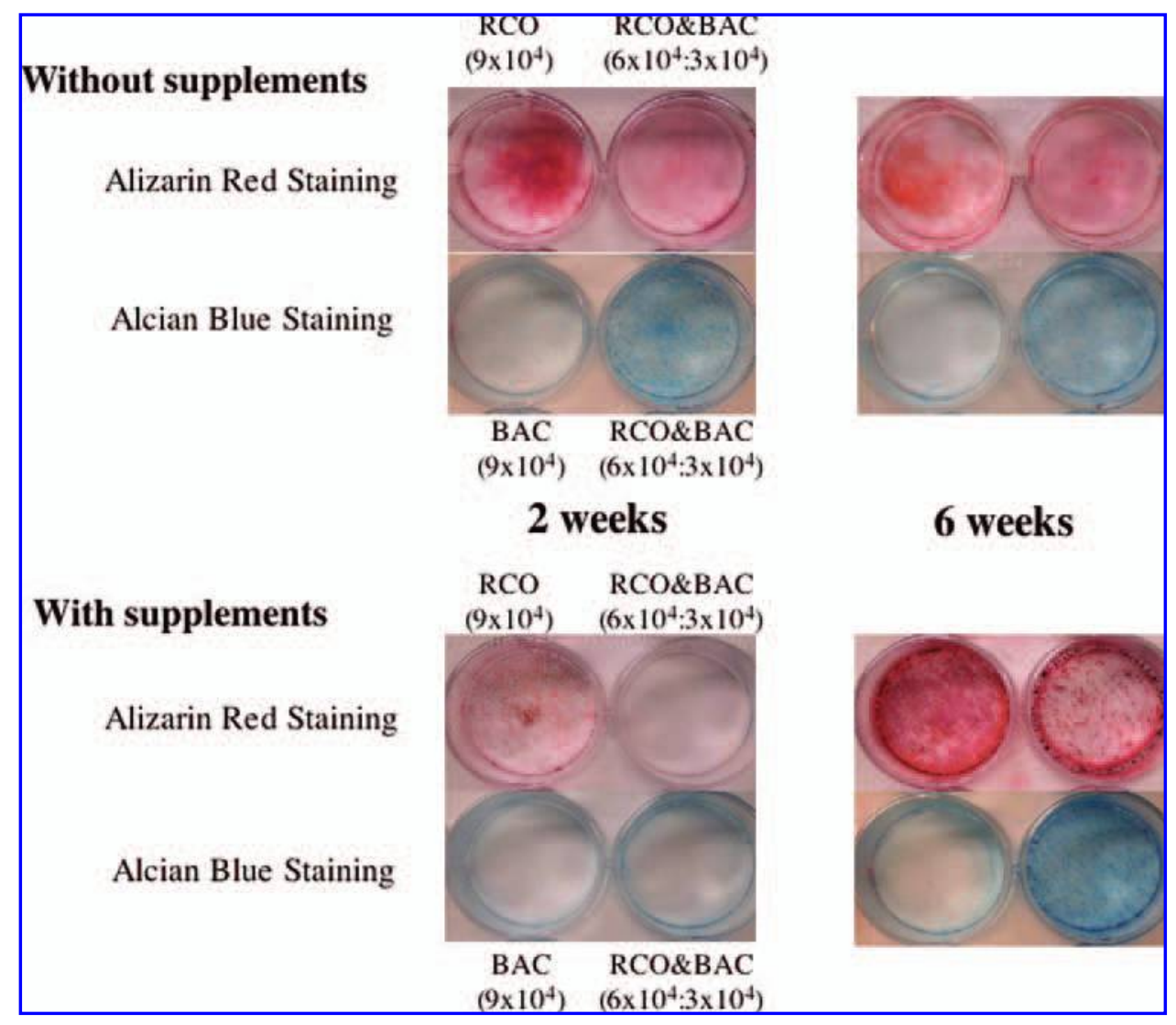

FIG. 1. Change in differentiation level of rat calvarial osteoblasts (RCOs) and bovine articular chondrocytes (BACs) by their coculture in alpha-minimum essential medium/Dulbecco's modified Eagle medium/F12 (1:1) containing 10\% fetal calf serum with or without osteogenic supplements [dexamethasone $(10 \mathrm{nM})$, beta-glycerophosphate $(10 \mathrm{mM})$, and ascorbic acid $(50 \mu \mathrm{g} / \mathrm{ml})$ ], which enhance osteoblast differentiation. Calcium deposited by RCOs and proteoglycan produced by BACs were qualitatively estimated using alizarin red staining and Alcian blue staining, respectively. Results from a single culture of each type at the same time point are shown in the left well. The number of each type on day 0 is indicated in the figure. 
comparisons and to compute the least significant difference between means.

\section{RESULTS}

\section{Direct co-culture studies}

RCOs and BACs were first mixed in a ratio of 2:1 and co-cultured in 12-well plates for 6 weeks. The effect of coculture was qualitatively analyzed using alizarin red and Alcian blue staining (Fig. 1). Alcian blue staining indicated that co-culture led to greater proteoglycan and glycosaminoglycan deposition after 2-week culture, even though the number of BACs on the starting day in co-culture was one third of the control group containing BACs only. These effects were maintained over time. The total cell number of RCO-alone culture, BAC-alone culture, and co-culture after 6-week culture were approximately $7.7 \times 10^{5}, 15.8 \times 10^{5}$, and $15.1 \times 10^{5}$, respectively. Similar to results obtained in the absence of osteogenic supplements, co-culture with osteogenic reagents again enhanced BAC differentiation. In contrast, no enhancement of RCO differentiation with co-culture was observed using alizarin red staining. Addition of osteogenic supplements enhanced RCO differentiation in solo culture, but again co-culture appeared to have no effect on the intensity of alizarin red staining. The total cell number of RCO-alone culture, BAC-alone culture, and co-culture after 6-week culture with the supplements was approximately $1.0 \times 10^{6}, 1.1 \times 10^{6}$, and $1.7 \times 10^{6}$, respectively.

The level of osteogenesis was next quantitatively analyzed for RCOs in co-culture and solo culture in the presence of osteogenic supplements. Figure 2 (A and B) shows the ALP activity and the production rate of osteocalcin, without normalization by cell number, in RCO solo culture

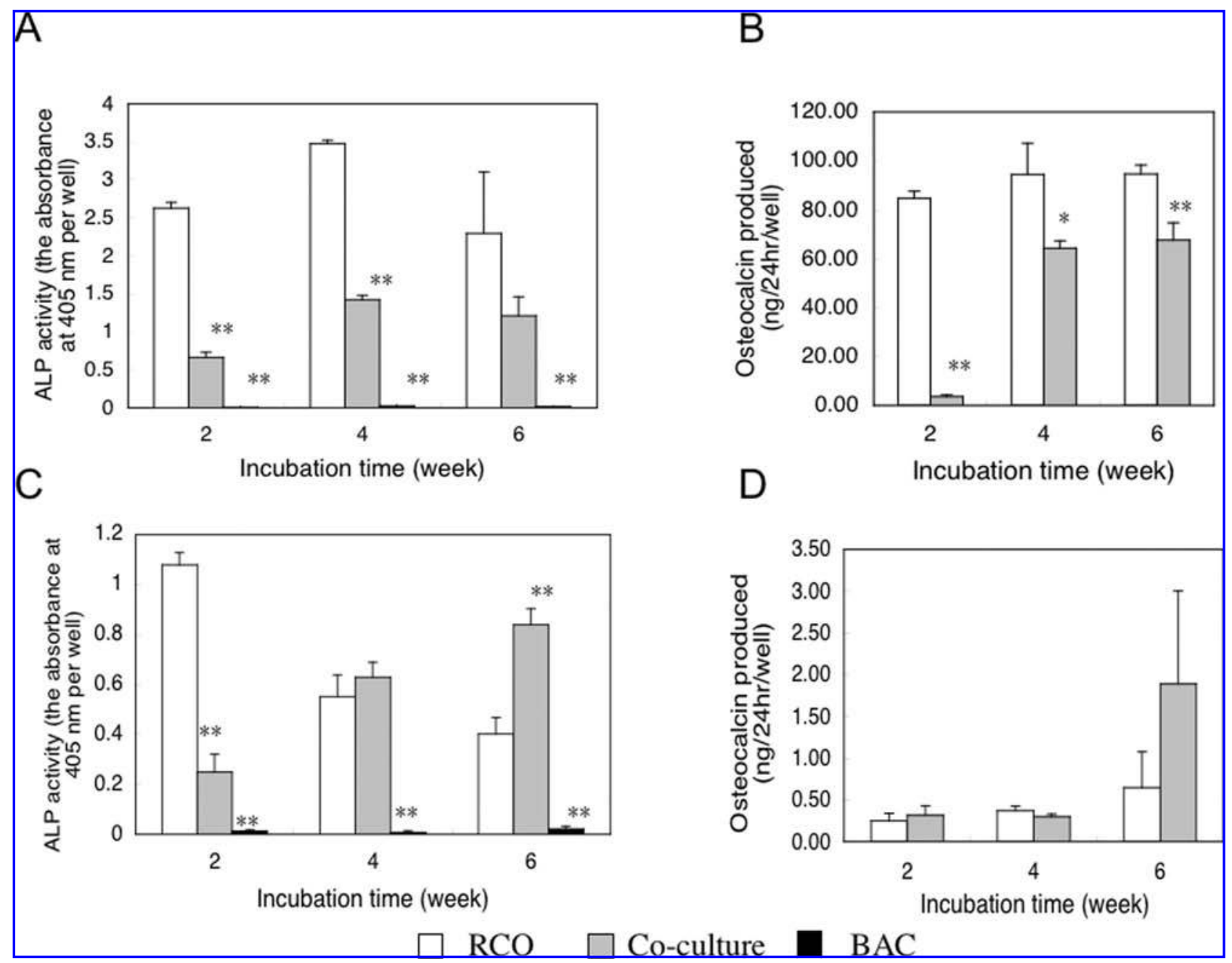

FIG. 2. Effect of co-culture with bovine articular chondrocytes (BACs) on rat calvarial osteoblast (RCO) differentiation, estimated from alkaline phosphatase activity expressed as the absorbance at $450 \mathrm{~nm}(\mathbf{A}, \mathbf{C})$ and the amount of osteocalcin secretion into the medium (B, D). RCOs (open column), BACs (closed column), and RCOs with BACs (gray column) were cultured in medium containing $10 \%$ fetal calf serum with the osteogenic supplements $(\mathbf{A}, \mathbf{B})$ or without the supplements $(\mathbf{C}, \mathbf{D})$. Data not normalized by the cell number, $* p<0.05, * * p<0.01$ against respective single $\mathrm{RCO}$ culture. 


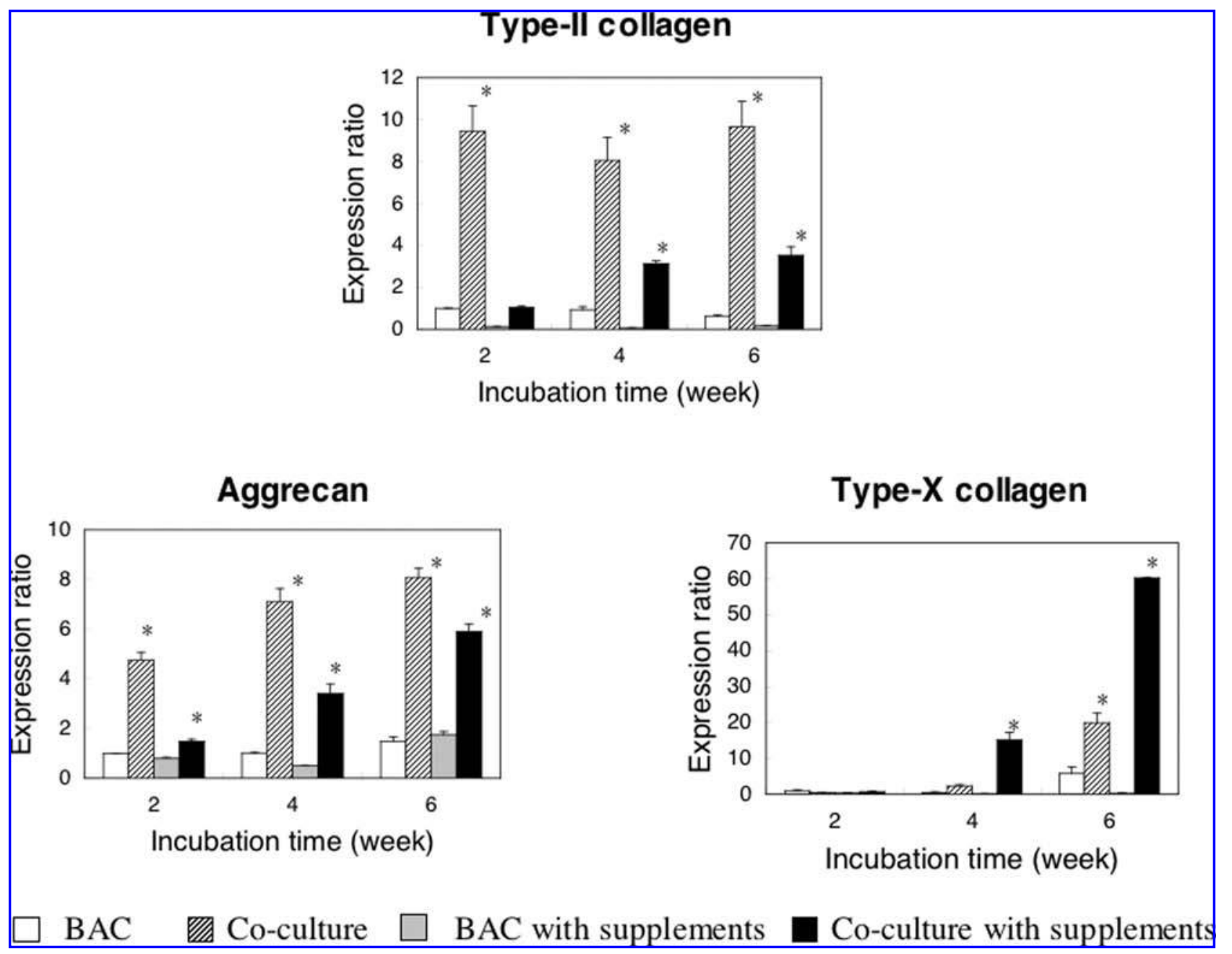

FIG. 3. Change in expression level of type-II collagen, aggrecan, and type-X collagen messenger ribonucleic acid (mRNA), which indicates the differentiation level of chondrocytes, during co-culture of bovine articular chondrocytes (BACs) with rat calvarial osteoblasts (RCOs). Cells were cultured in medium with or without the osteogenic supplements (open column: BACs alone without the supplements; striped column: co-cultured BACs without the supplements; gray column: BACs alone with the supplements; closed column: co-cultured BACs with the supplements). All data were normalized using the expression level of glyseraldehyde-3-phosphate dehydrogenase mRNA and expressed as a ratio of the expression level in the BAC-alone culture at 2 weeks without the osteogenic supplements $(* p<0.01$ against BAC-alone culture under the same condition).

and co-culture with BACs with osteogenic supplements. The ALP activity detected in the RCO-alone culture was always greater than that in co-culture system, irrespective of incubation time (Fig. 2A). The magnitude of these effects appears reasonable, because the RCO number in the co-culture is likely to be less than that in RCO-alone culture, as based on initial seeding densities. No measurable ALP activity was noted in BAC-alone cultures. Similar to the ALP activity, osteocalcin production from the co-culture system was less than with RCO-alone cultures. However, the amount of osteocalcin production in the co-culture condition markedly and significantly increased after 4 weeks (Fig. 2B, $p<0.01$ against co-culture group at 2 weeks).

Quite different results on RCO differentiation were noted when these analyses were performed on cells cultured without osteogenic supplements. ALP activity in the coculture condition increased over time, whereas ALP decreased over time in RCO-alone culture, and ALP activities were significantly greater in co-culture at the later time points (Fig. 2C). Again, minimal ALP activity was noted in BAC-alone cultures. The osteocalcin production from RCOs in co-culture or single culture increased with time but was low even after a 6-week incubation (Fig. 2D). Coculture of RCOs with BACs led to a 3-fold higher osteocalcin secretion rate at the last time point, although this difference was not significant (Fig. 2D).

Total RNA from the co-culture was also extracted, and the expression of mRNA for type-II and type-X collagen and aggrecan was analyzed with or without osteogenic supplements. When the cells were cultured without the osteogenic supplements, mRNA for type-II collagen and 
Table 2. Effect of 4-Week Co-Culture of Rat Calvarial Osteoblasts (RCOs) and Bovine Articular Chondrocytes (BACs) on Cell Number and Alkaline Phosphatase Activity of RCOs Using Transwell Culture System to Avoid Their Direct Contact

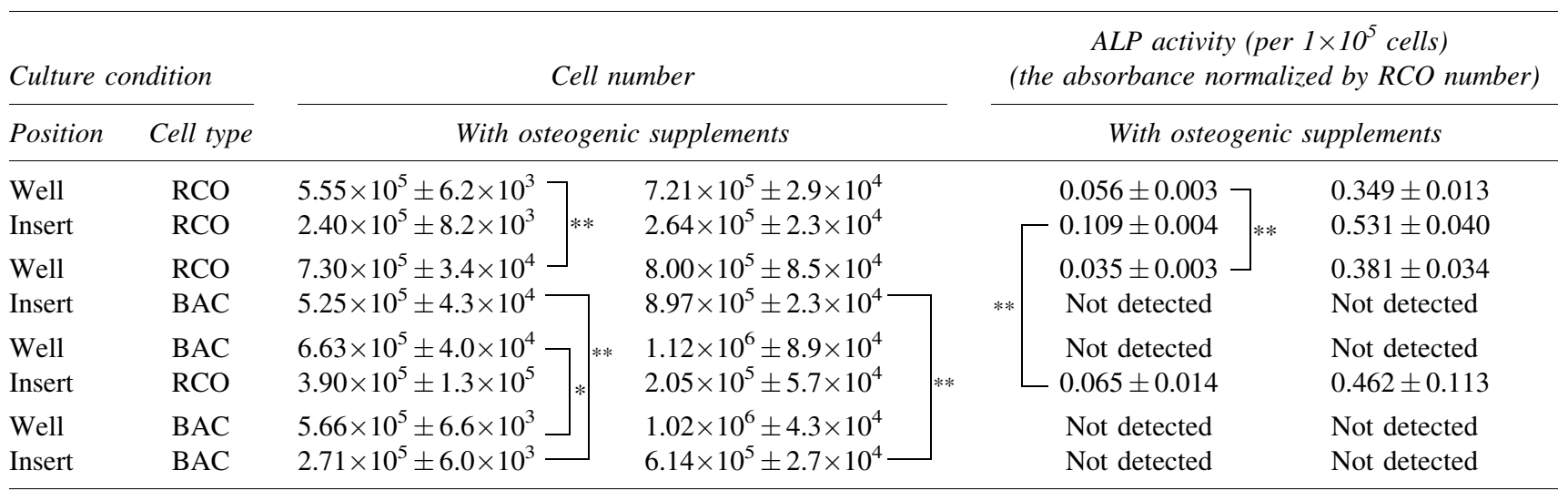

$* p<0.05, * * p<0.01$.

aggrecan were markedly enhanced in co-culture with RCOs, even after only 2 weeks. In BAC-alone cultures, aggrecan and type-X collagen mRNA expression in BAC slightly increased after a 6-week incubation, whereas type-II collagen mRNA expression was maintained at a low level during the experimental period. A similar enhancement of BAC differentiation with co-culture was noted when cells were maintained in medium containing osteogenic supplements, although the magnitude of BAC differentiation, as measured according to type-II collagen and aggrecan expression, decreased (Fig. 3). Expression of type-X collagen mRNA was significantly enhanced after 4 to 6 weeks in this condition.

Additional studies indicated that there were direct GJICs between RCOs and BACs (data not shown), even though the 2 cell types were isolated from different species, suggesting that the RCOs and BACs might influence each other through this mode of communication.

\section{Co-culture using transwell system}

In transwell co-culture experiments that allow cells to share medium without direct contact, the number of RCOs and BACs increased more than with a single-cell culture after a 4-week incubation (Table 2). With osteogenic supplements, however, the increase in the number of cocultured RCOs was not observed, and the increase in the number of co-cultured BACs was smaller than the increase in co-cultured BACs without the supplements. When cultures were stained using Alcian blue after a 4-week incubation, co-cultured BACs in the presence or absence of osteogenic supplements were stained more intensely than BAC-alone cultures under the same culture condition (Fig. 4A). Quantification of the Alcian blue dye extracted from cultures demonstrated that more bound dye per BAC was always present in co-cultures, but addition of osteogenic supplements suppressed proteoglycan deposition (Fig. 4B). Co-culture in the absence of osteogenic supplements in the transwell system did not enhance RCO differentiation. The
RCO ALP level in co-culture normalized by cell number was approximately two-thirds that in RCO-alone culture (Table 2). When the osteogenic supplements were added to the medium, however, the alizarin red stained co-cultured RCOs more intensely than the RCO-alone culture (Fig. 4A). Moreover, ALP activity in co-culture and RCO-alone culture was similar in this condition (Table 2). The same results were observed in these experiments regardless of which cell type was placed in the bottom well or insert of the transwell system (data not shown).

\section{DISCUSSION}

Because it was previously reported that a growth platelike tissue was regenerated when arginine-glycine-aspartic acid (RGD)-modified alginate gel discs containing a simple mixture of RCOs and BACs (ratio of 2:1) were implanted into SCID mice for 26 weeks, ${ }^{4}$ unknown interaction between RCOs and BACs might play an important role in this tissue regeneration. In vitro co-culture experiments in the current study indicated that co-culture of BACs with RCOs enhanced BAC proliferation and differentiation levels via direct contact with RCOs and paracrine effects of soluble factors secreted from the RCOs and that the phenotype of the co-cultured RCOs influences the level of this enhancement. In addition, BACs regulate co-cultured RCOs, although the effects are lower in magnitude.

Simple, direct co-culture of RCOs and BACs increased the total cell number in the culture 1.5 to 2 times as much as RCO- or BAC-alone (only when osteogenic supplements were added) culture, although the contribution of each cell type to the total cell number was not assessed. However, transwell experiments (Fig. 4) suggested that the number of RCOs and BACs increased in co-culture. The mechanism of this effect must be clarified in future studies.

The results of this study indicated that not only cell proliferation but also cell differentiation was enhanced 
using co-culture of chondrocytes and osteoblasts. BAC differentiation level was first estimated from Alcian blue staining (Fig. 1). Because Alcian blue molecules bind to proteoglycans, ${ }^{17-19}$ the BAC differentiation level can be estimated from the density of Alcian blue binding to proteoglycans that are secreted from differentiated BACs. The magnitude of this effect in the co-cultures depended on whether osteogenic supplements were added to the medium, indicating that the differentiation level of the cocultured RCOs influenced the BAC differentiation level. When mRNA levels of type-II collagen, aggrecan, and type-X collagen, which can be used as markers indicating chondrocyte differentiation, ${ }^{21,22}$ were measured using realtime PCR, the expression of type-II collagen and aggrecan in BAC decreased with addition of the osteogenic supplements (Fig. 3). When BACs were co-cultured with RCO without the supplements, mRNA of type-II collagen and aggrecan were expressed 5 to 9 times as higher as those expressed in a single BAC culture. When these mRNA expression levels were quantified in BAC and RCO coculture with the supplements, the levels were lower than the co-culture without the supplements. Alternatively, type-X collagen was strongly expressed in the co-culture with the supplements, and the level in this condition was 60 times as high as the single BAC culture and 3 times as high as the co-culture without the supplements. These findings suggest that co-culture of BACs with osteogenic supplements leads to differentiation into a hypertrophic phenotype ${ }^{23}$ and indicate that the differentiation level of the co-cultured RCOs regulates the differentiation stage of the co-cultured BACs. The studies using transwell culture (Fig. 4) further suggested that soluble factors produced by RCOs are at least partially responsible for the enhanced BAC proliferation and differentiation level in co-culture, because there is no direct contact between RCOs and BACs in this condition. It has been previously demonstrated that many growth factors, including the TGFs, FGFs, and IGFs, play a role in chondrocyte differentiation. ${ }^{5-8}$ One or two of these soluble factors may be secreted from RCOs to induce BAC differentiation. In addition, some components in FCS may have an additional effect on the BAC differentiation in concert with the cell-secreted soluble factors. The soluble factors that mediate these effects remain to be identified.

Enhancement of RCO differentiation was not observed with co-culture with BACs when analyzed using alizarin red staining (Fig. 1). Although one probable reason for this result was ascribed to the initially different RCO number in the co-culture, the ALP activity after 2-week culture was still lower than what would be expected based on the initial seeding density of the RCOs. The ALP activity was also suppressed in co-culture using the Transwell system even after 4-week culture (Table 2). This suggests the co-culture

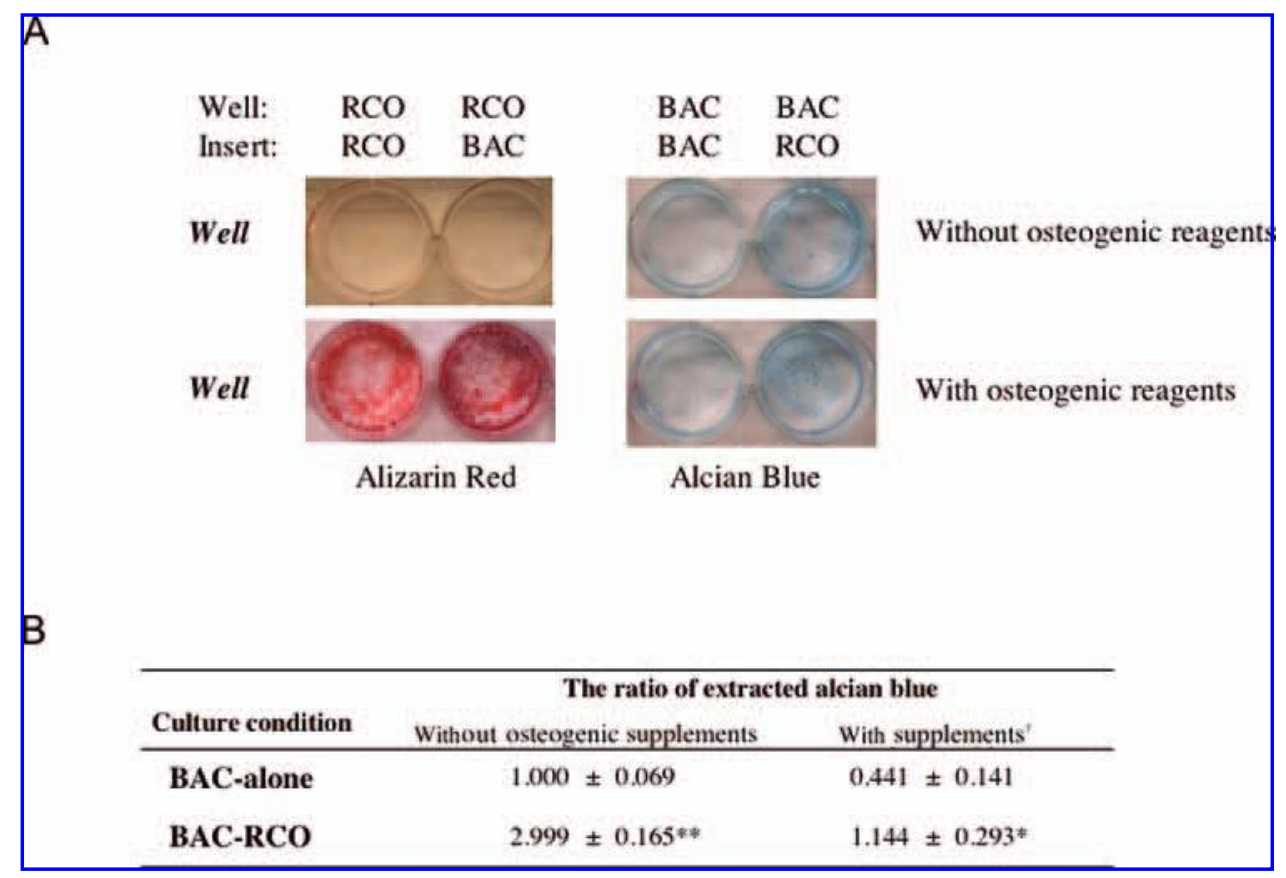

FIG. 4. Effect of rat calvarial osteoblasts (RCOs) and bovine articular chondrocytes (BACs) co-culture on their differentiation using a transwell system to avoid direct contact between the 2 cell types. RCOs and BACs were separately cultured for 4 weeks with or without the osteogenic supplements. The differentiation level of RCOs and BACs in the bottom wells of the system were estimated using alizarin red and Alcian blue staining, respectively (A). To determine the differentiation level of BACs, proteoglycan-bound Alcian blue dye was extracted, and the absorbance of the extract was expressed as a ratio of the value obtained from BAC-BAC transwell culture without the osteogenic supplements. All data were normalized using BAC number (per $1 \times 10^{5}$ cells) $(\mathbf{B})(* p<0.05$, $* * p<0.01$ against BAC-BAC culture in the same condition. ${ }^{\dagger} p<0.01$ against the same co-culture combination without osteogenic supplements). 
with BACs suppresses the differentiation of the RCOs in the early stages. However, the ALP activity of the cells in co-culture without the supplements increased as their incubation time increased, whereas the activity of singleRCO culture decreased over time. Because BACs appeared to differentiate to a hypertrophic phenotype with time (type-X collagen mRNA expression increased as incubation time increased), this ALP increase in the co-culture may be ascribed to an increase in ALP production from the hypertrophic chondrocytes. However, the osteocalcin production was increased after 6-week co-culture, although its value was still less than the values found in co-culture with the supplements (Fig. 2C, D). In addition, it has been suggested that hypertrophic chondrocytes regulate endochondral ossification. ${ }^{23,24}$ Therefore, these findings suggest that co-culture may enhance some measures of in vitro RCO differentiation but not all. Expression of type-I collagen mRNA in solo culture and co-culture should be examined in the future to analyze the effect of co-culture on RCO differentiation in more detail, as well as to further analyze the differentiation level of the BACs. Alternatively, when ALP activity and osteocalcin production from the coculture with osteogenic supplements were measured, both values were less than those from $\mathrm{RCO}$-alone culture, irrespective of incubation time (Fig. 2A, B). These results suggest that maximal enhancement of RCO differentiation may be obtained with the supplements alone.

When results from simple co-culture of RCOs and BACs were compared with those from their co-culture using the transwell system, some inconsistencies between the results could be observed. For example, Figs. 1, 2A, and 2B suggest suppression in differentiation of RCOs co-cultured with BACs and osteogenic supplements, whereas results from transwell culture with supplements (Table 2 and Fig. 4) indicate no suppression in their differentiation. Similarly, BACs co-cultured with RCOs and the supplements in the transwell system has decreased deposition of proteoglycans, although simple co-culture with the supplements enhanced deposition of proteoglycans. These inconsistencies between direct and transwell co-culture could result from direct interactions (e.g., GJIC) that differentially regulate the phenotype of each cell type. The RCOs and BACs used in these studies were derived from different species, rat and bovine, and it was expected that there would be no direct interaction between these cell types. When GJIC, which is known to regulate homeostasis in cells and tissues ${ }^{25-27}$ was estimated, communication between RCOs and BACs was observed (data not shown). Therefore, in addition to unidentified soluble factors, this communication and other direct interactions may play a role in controlling BAC and $\mathrm{RCO}$ differentiation in the co-culture system and probably in previous in vivo experiments. Clarifying these various types of cross talk between RCOs and BACs, as well as cross talk between these cells and stem cells in the future may be essential to clinically regenerating growth plate tissue effectively.
The results of this study indicate direct, reciprocal instructive interactions between co-cultured osteoblasts and chondrocytes. Therefore, it may be necessary to account for this signaling to regenerate complex tissues composed of several kinds of tissues and a mixture of various cell types, including stem cells. Similar studies using human cells will also be valuable to validate the importance of this work for human disease and regeneration.

\section{ACKNOWLEDGMENTS}

Ryusuke Nakaoka and this work were supported by an overseas assignment program for Japanese Researchers from the Japan Health Sciences Foundation (Health and Labour science research grants for research on health sciences focusing on drug innovation, Ministry of Health, Labour and Welfare, Japan) and dispatch of a Japanese researcher to a foreign country by the Japan Association for the Advancement of Medical Equipment (Health and Labour science research grants for research on advanced medical technology, Ministry of Health, Labour and Welfare, Japan). Ryusuke Nakaoka would like to thank Dr. Toshie Tsuchiya (NIHS, Japan) for her support in obtaining this funding.

\section{REFERENCES}

1. Mooney, D.J., and Mikos, A.G. Growing new organs. Sci. Am. 280, 38, 1999.

2. Oberpenning, F., Meng, J., Yoo, J.J., and Atala, A. De novo reconstitution of a functional mammalian urinary bladder by tissue engineering. Nat. Biotech. 17, 149, 1999.

3. Humes, H.D., Buffington, D.A., MacKay, S.M., Funke, A.J., and Weitzel, W.F. Replacement of renal function in uremic animals with a tissue engineered kidney. Nat. Biotech. 17, 451, 1999.

4. Alsberg, E., Anderson, K.W., Albeiruti, A., Rowley, J.A., and Mooney, D.J. Engineering growing tissues. Proc. Natl. Acad. Sci. U. S. A. 99, 12025, 2002.

5. Van der Eerden, D.C.J., Karperien, M., and Wit, J.M. Systemic and local regulation of the growth plate. Endocr. Rev. 24, 782, 2003.

6. Grimsrud, C.D., Romano, P.R., D'Souza, M., Puzas, J.E., Reynolds, P.R., Rosier, R.N., and O'Keefe, R.J. BMP-6 is an autocrine stimulator of chondrocyte differentiation. J. Bone Miner. Res. 14, 475, 1999.

7. Tuli, R., Tuli, S., Nandi, S., Huang, X., Manner, P.A., Hozack, W.J., Danielson, K.G., Hall, D.J., and Tuan, R.S. Transforming growth factor-beta-mediated chondrogenesis of human mesenchymal progenitor cells involves $\mathrm{N}$-cadherin and mitogen-activated protein kinase and Wnt signaling cross-talk. J. Biol. Chem. 278, 41227, 2003.

8. Ionescu, A.M., Schwarz, E.M., Zuscik, M.J., Drissi, H., Puzas, J.E., Rosier, R.N., and O'Keefe, R.J. ATF-2 cooperates with Smad3 to mediate TGF-beta effects on chondrocyte maturation. Exp. Cell Res. 288, 198, 2003. 
9. Wozney, J.M. Bone morphogenetic proteins. Prog. Growth Factor Res. 1, 267, 1989.

10. Noda, M., and Camilliere, J.J. In vivo stimulation of bone by transforming growth factor-beta. Endocrinology 124, 2991, 1989.

11. Horner, A., Kemp, P., Summers, C., Bord, S., Bishop, N.J., Kelsall, A.W., Coleman, N., and Compston, J.E. Expression and distribution of transforming growth factor-beta isoforms and their signaling receptors in growing human bone. Bone 23, 95, 1998.

12. Martin, G.R. The roles of FGFs in the early development of vertebrate limbs. Gene Dev. 12, 1571, 1998.

13. Franceschi, R.T., Xiao, G., Jiang, D., Gopalakrishnan, R., Yang, S., and Reith, E. Multiple signaling pathways converge on the Cbfa1/Runx2 transcription factor to regulate osteoblast differentiation. Connect. Tissue Res. 44(Suppl. 1), 109, 2003.

14. Gerstenfeld, L.C., Barnes, G.L., Shea, C.M., and Einhorn, T.A. Osteogenic differentiation is selectively promoted by morphogenetic signals from chondrocytes and synergized by a nutrient rich growth environment. Connect. Tissue Res. 44(Suppl. 1), 85, 2003.

15. Ohyama, M., Suzuki, N., Yamaguchi, Y., Maeno, M., Otsuka, K., and Ito, K. Effect of enamel matrix derivative on the differentiation of C2C12 cells. J. Periodontol. 73, 543, 2002.

16. Isama, K., and Tsuchiya, T. Enhancing effect of poly(L-lactic) acid on the differentiation of mouse osteoblasts-like MC3T3E1 cells. Biomaterials 24, 3303, 2003.

17. Rahman, M.S., and Tsuchiya, T. Enhancement of chondrogenic differentiation of human articular chondrocytes by biodegradable polymers. Tissue Eng. 7, 781, 2001.

18. Whiteman, P. The quantitative measurement of Alcian blueglycosaminoglycan complexes. Biochem. J. 131, 343, 1973.

19. Terry, D.E., Chopra, R.K., Ovenden, J., and Anastassiades, T.P. Differential use of alcian blue and toluidine blue dyes for quantification and isolation of anionic glycoconjugates from cell culture: application to proteoglycans and high-molecularweight glycoprotein synthesized by articular chondrocytes. Anal. Biochem. 285, 211, 2000.

20. Dürig, J., Rosental, C., Halfmeyer, K., Wiemann, M., Novotny, J., Bingmann, D., Dührsen, U., and Schirrmmacher, K.
Intercellular communication between bone marrow stromal cells and CD34+ haematopoietic progenitor cells is mediated by connexin 43-type gap junctions. Br. J. Haematol. 111, 416, 2000.

21. Olsen, B.R. New insights into the function of collagens from genetic analysis. Curr. Opin. Cell Biol. 7, 720, 1995.

22. Doege, K., Sasaki, M., Horigan, E., Hassel, J.R., and Yamada, Y. Complete primary structure of the rat cartilage proteoglycan core protein deduced from cDNA clones. J. Biol. Chem. 262, 17757, 1987.

23. Iyama, K., Ninomiya, Y., Olsen, B.R., Linsenmayer, T.F., Trelstad, R.L., and Hayashi, M. Spatiotemporal pattern of type $\mathrm{X}$ collagen gene expression and collagen deposition in embryonic chick vertebrae undergoing endochondral ossification. Anat. Rec. 229, 462, 1991.

24. Nagamoto, N., Iyama, K., Kitaoka, M., Ninomiya, Y., Yoshioka, H., Mizuta, H., and Takagi, K. Rapid expression of collagen type $\mathrm{X}$ gene of non-hypertrophic chondrocytes in the grafted chick periosteum demonstrated by in situ hybridization. J. Histochem. Cytochem. 41, 679, 1993.

25. Mensil, M., Krutovskikh, V., Omori, Y., and Yamasaki, H. Role of blocked gap junctional communication in nongenotoxic carcinogenesis. Toxicol. Lett. 82/83, 701, 1995.

26. Yamasaki, H. Role of disrupted gap junctional intercellular communication in detection and characterization of carcinogens. Mutat. Res. 365, 91, 1996.

27. Maio, A.D., Vega, V.L., and Contreras, J.E. Gap junctions, homeostasis, and injury. J. Cell Physiol. 191, 269, 2002.

Address reprint requests to: Ryusuke Nakaoka, Ph.D.

Division of Medical Devices National Institute of Health Sciences 1-18-1 Kamiyoga, Setagaya-ku Tokyo 158-8501, Japan

E-mail: nakaoka@nihs.go.jp 



\section{This article has been cited by:}

1. Prof. Frank Luyten , Petros Lenas , Prof. Malcolm Jr Moos . Developmental Engineering: A new paradigm for the design and manufacturing of cell based products. Part I: From three-dimensional cell growth to biomimetics of in vivo developmentDevelopmental Engineering: A new paradigm for the design and manufacturing of cell based products. Part I: From three-dimensional cell growth to biomimetics of in vivo development. Tissue Engineering Part B: Reviews 0:ja. . [Abstract] [PDF] [PDF Plus]

2. Andrew D. Thompson, Martha W. Betz, Diana M. Yoon , John P. Fisher . 2009. Osteogenic Differentiation of Bone Marrow Stromal Cells Induced by Coculture with Chondrocytes Encapsulated in Three-Dimensional MatricesOsteogenic Differentiation of Bone Marrow Stromal Cells Induced by Coculture with Chondrocytes Encapsulated in Three-Dimensional Matrices. Tissue Engineering Part A 15:5, 1181-1190. [Abstract] [PDF] [PDF Plus]

3. Sylvie Miot, Roberto Gianni-Barrera, Karoliina Pelttari, Chitrangada Acharya , Pierre Mainil-Varlet, Henriette Juelke , Claude Jaquiery, Christian Candrian, Andrea Barbero, Ivan Martin. In Vitro and In Vivo Validation of Human and Goat Chondrocyte Labeling by Green Fluorescent Protein Lentivirus TransductionIn Vitro and In Vivo Validation of Human and Goat Chondrocyte Labeling by Green Fluorescent Protein Lentivirus Transduction. Tissue Engineering Part C: Methods, ahead of print. [Abstract] [PDF] [PDF Plus]

4. Jian Li , Shobha Mareddy, Dawn Meifang Tan, Ross Crawford, Xing Long, Xigeng Miao , Yin Xiao . A Minimal Common Osteochondrocytic Differentiation Medium for the Osteogenic and Chondrogenic Differentiation of Bone Marrow Stromal Cells in the Construction of Osteochondral GraftA Minimal Common Osteochondrocytic Differentiation Medium for the Osteogenic and Chondrogenic Differentiation of Bone Marrow Stromal Cells in the Construction of Osteochondral Graft. Tissue Engineering Part $A$, ahead of print. [Abstract] [PDF] [PDF Plus] 